\title{
Effect of Metformin on Lactate Metabolism in Normal He- patocytes under High Glucose Stress in Vitro
}

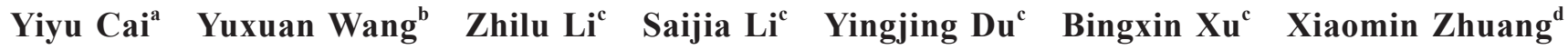 \\ Guanlu Li ${ }^{\mathrm{e}}$ Daifeng Zhou ${ }^{\mathrm{f}}$
}

Hainan Medical University a.College of Tropical Medicine and Laboratory Medicine; b.College of Public Health; c.The First Clinical College; d.College of Management; e.College of International Education; f.College of Basic Medicine and Life Sciences, Haikou, Hainan, 570100, China

\begin{abstract}
Objective:To study the effect of metformin on lactate metabolism in hepatocytes in vitro under high glucose stress. Methods:LO2 hepatocytes were cultured in vitro, and cells were randomly divided into blank control, $25 \mathrm{mmol} / \mathrm{L}$ glucose solution, $27 \mathrm{mmol} / \mathrm{L}$ glucose solution, $29 \mathrm{mmol} / \mathrm{L}$ glucose solution, $31 \mathrm{mmol} / \mathrm{L}$ glucose solution, $33 \mathrm{mmol} / \mathrm{L}$ glucose solution, $35 \mathrm{mmol} / \mathrm{L}$ glucose solution treatment groups, and then into blank control groups after determining the optimal concentration of $31 \mathrm{mmol} / \mathrm{L}$ and use $30 \mathrm{mmol} / \mathrm{L} \mathrm{metformin}$ solution, and then divided into blank control group. The optimal concentration of glucose solution, normal liver cells + metformin solution normal liver cells, the optimal concentration of glucose solution normal liver cells +metformin solution respectively in the $12 \mathrm{~h}, 24$ $\mathrm{h}, 48 \mathrm{~h}$ on cell count plate to calculate the number of liver cells, and using lactic acid determination kit the optimal concentration of glucose solution+normal liver cells and normal liver cells+the optimal concentration of glucose solution+metformin solution respectively in the $12 \mathrm{~h}, 24 \mathrm{~h}, 48 \mathrm{~h}$ of cell cultures of lactic acid value. Results: There was no significant change in the lactic acid concentration but significant increase in the number of surviving hepatocytes in the high-glycemic control group compared with that in the high-glycemic control group without metformin. Conclusion:Metformin has no significant effect on lactic acid metabolism of hepatocytes under high glucose stress in vitro, and has a protective effect on hepatocytes under high glucose stress. Based on this, it is preliminarily believed that metformin is not the direct factor leading to diabetic lactic acidosis.
\end{abstract}

\section{Keywords}

metformin; liver cells; metformin associated lactic acidosis (MALA)

\section{二甲双胍对体外高糖胁迫下正常肝细胞内乳酸代谢的影响}

\author{
蔡奕渔 ${ }^{a}$ 王宇萱 ${ }^{b}$ 李志路 ${ }^{c}$ 李赛嘉 ${ }^{c}$ 杜荣婧 ${ }^{c}$ 许冰心 ${ }^{c}$ 庄小敏 ${ }^{d}$ 黎冠陆 $^{e}$ 周代锋 ${ }^{{ }^{*}}$ \\ 海南医学院 a. 热带医学与检验医学院； b. 公共卫生学院； c. 第一临床学院； d. 管理学院； e. 国际教育学院；f. 基础医学与生命 \\ 科学学院, 中国・海南海口 570100
}

\section{摘 要}

目的: 研究二甲双胍对高糖胁迫下的体外肝细胞产生乳酸代谢的影响。方法: 体外培养 LO2 肝细胞, 细胞随机分为空白对照 组, $25 \mathrm{mmol} / \mathrm{L}$ 葡萄糖溶液、 $27 \mathrm{mmol} / \mathrm{L}$ 葡萄糖溶液、 $29 \mathrm{mmol} / \mathrm{L}$ 葡萄糖溶液、 $31 \mathrm{mmol} / \mathrm{L}$ 葡萄糖溶液、 $33 \mathrm{mmol} / \mathrm{L}$ 葡萄糖溶液、 $35 \mathrm{mmol} / \mathrm{L}$ 葡萄糖溶液处理组, 确定最佳浓度为 $31 \mathrm{mmol} / \mathrm{L}$ 后, 使用 $30 \mathrm{mmol} / \mathrm{L}$ 二甲双胍溶液, 然后分为空白对照组。正常肝 细胞 + 最适浓度葡萄糖溶液、正常肝细胞 + 二甲双胍溶液、正常肝细胞 + 最适浓度葡萄糖溶液 + 二甲双胍溶液分别在 $12 \mathrm{~h} 、$ $24 \mathrm{~h} 、 48 \mathrm{~h}$ 时用细胞计数板计算出肝细胞的数量, 并运用乳酸试剂盒测定正常肝细胞 + 最适浓度葡萄糖溶液和正常肝细胞 + 最适浓度葡萄糖溶液 + 二甲双胍溶液分别在 $12 \mathrm{~h} 、 24 \mathrm{~h} 、 48 \mathrm{~h}$ 时的细胞培养液的乳酸值。结果: 与未加入二甲双胍的高糖胁迫 肝细胞实验组相比, 加入二甲双胍的高糖胁迫肝细胞实验组的乳酸浓度无显著变化, 但存活的肝细胞数目显著增加。结论: 二甲双胍对高糖胁迫下的体外肝细胞产生乳酸代谢无明显影响，且对高糖胁迫下的肝细胞具有一定的保护作用。基于此，初 步认为二甲双胍并不是导致糖尿病患者相关性乳酸酸中毒的直接因素。

\section{关键词}

二甲双胍; 肝细胞; 二甲双胍相关性乳酸酸中毒（MALA）

\section{1 引言}

随着人们生活方式和习惯的改变, 糖尿病的发病率呈直
在 2025 年达到 5900 万人，其中有 $90 \%$ 以上的患者为 II 型糖 尿病 $(\mathrm{T} 2 \mathrm{MD})^{[1-3]}$, 中国是糖尿病患者人数最多的国家 ${ }^{[4]}$ 。

因糖尿病发生发展涉及多种因素，发病机制较为复杂，而且 
降糖药物在控制血糖和延缓并发症发生方面发挥了不错的作 用，但是仍然存在一些局限性及不良反应。

二甲双胍是一种双胍类口服抗糖尿病药物, 因其具有 显著的降糖作用及多方面的临床应用价值, 在临床中被广 泛应用, 国际上也将二甲双胍作为首选的降糖药物。 $\mathrm{HOME}$ 和 DDP 实验研究表明了二甲双胍对糖尿病治疗的安全性和 有效性 ${ }^{[5]}$ 。然而, 随着临床上二甲双胍的应用范围增大, 在其作为降糖药的使用过程中也逐渐显示出一些副作用, 其中乳酸酸中毒 ( LA ) 是临床医生一直担忧的问题。患者 服用二甲双胍引起的乳酸酸中毒, 称为二甲双胍相关性乳 酸酸中毒 (MALA), MALA 是使用二甲双胍治疗过程中 出现的少见且严重的不良反应, 是由于二甲双胍阻碍了线 粒体中乳酸向葡萄糖转化的通路, 引起体内乳酸生成过多 或清除过少, 导致机体产生了代谢性疾患, 这种疾病的致 死率非常高 ${ }^{[6-7]}$ 。

有研究表明二甲双胍导致乳酸酸中毒可能与糖尿病患 者自身具有严重疾病有关 ${ }^{[8]}$, 其中主要与患者肾功能不全 心功能不全、缺氧状态等有联系。基于此, 论文针对二甲双 胍干预下体外高糖胁迫下正常肝细胞乳酸代谢的影响进行阐 述，进而探索二甲双胍对乳酸的代谢是否具有直接的关联 性, 为临床上治疗糖尿病以及二甲双胍的合理使用提供参考 依据。

\section{2 材料与方法}

\section{1 主要试剂与仪器}

试剂: 细胞选取 LO2 肝细胞株、DMEM 培养基、胰酶、 青霉素一链霉素双抗混合溶液、二甲双胍、10\%胎牛血清、 乳酸测定试剂盒（均购于海口锐科生物科技有限公司）。

仪器: 显微镜、超净生物台、 $\mathrm{CO}_{2}$ 细胞培养箱。

\section{2 实验方法}

\section{2 .1 细胞培养}

LO2 细胞使用 $10 \%$ 胎牛血清的 DMEM 完全培养液, 于

【基金项目】大学生创新创业省级训练项目 (项目编号 : S201911810055)。

【第一作者】蔡奕渔, 男, 本科学历, 通讯邮箱: 735786405 @aq.com。

【通讯作者】周代锋, 男, 教授, 从事疾病发生的分子遗传机 制研究。 $37^{\circ} \mathrm{C} 、 5 \% \mathrm{CO}_{2}$ 培养箱中培育, 培养至细胞贴壁, 进行常规的 细胞培养, 1 2 天换液一次,当培养细胞覆盖率达 80\% 90\%时， 进行传代培养, 重复操作, 将一瓶即将传代的细胞进行冻存, 以免发生意外导致缺少 LO2 肝细胞无法进行实验。

\subsection{2 分组模型建立对照实验}

(1) 高糖模型的建立

本实验开始前进行了高糖对 LO2 肝细胞的预处理, 进 行了葡萄糖 ( Glucose, $G$ ) 最适宜浓度实验, G 的浓度梯 度设为 $0 \mathrm{mmol} / \mathrm{L}, 25 \mathrm{mmol} / \mathrm{L}, 27 \mathrm{mmol} / \mathrm{L}, 29 \mathrm{mmol} / \mathrm{L}, 31$ $\mathrm{mmol} / \mathrm{L}, 33 \mathrm{mmol} / \mathrm{L}$, 预实验得出 $31 \mathrm{mmol} / \mathrm{L}$ 作为最适实验 浓度, 并运用乳酸试剂盒测算孚酸含量。

\section{(2) 二甲双胍模型的建立}

本实验使用盐酸二甲双胍药片稀释至 $30 \mathrm{mmol} / \mathrm{L}$ 至细胞 培养基, 进行二甲双胍对 LO2 肝细胞的预处理, 培养时间为 $12 \mathrm{~h} 、 24 \mathrm{~h}$ 和 $48 \mathrm{~h}$ 。

\section{（3）干预模型的建立}

本实验将浓度为 $30 \mathrm{mmol} / \mathrm{L}$ 的盐酸二甲双胍培养液和浓 度为 $31 \mathrm{mmol} / \mathrm{L}$ 的葡萄糖溶液加入培养瓶，培养 $12 \mathrm{~h} 、 24 \mathrm{~h}$ 和 $48 \mathrm{~h}$ 后运用乳酸试剂盒测算乳酸含量。

\subsection{3 细胞计数法测定肝细胞增殖情况}

用胰酶将细胞分解，消化一段时间后加入培养液终止消 化, 再用移液枪移取培养液至计数板, 严格按照细胞计数规 则计数细胞。

\subsection{4 乳酸试剂盒法检测样本的乳酸情况}

在加入二甲双胍溶液和高糖处理的细胞溶液放人二氧化 碳细胞培养箱中培养 $12 \mathrm{~h} 、 24 \mathrm{~h}$ 和 $48 \mathrm{~h}$, 并分别运用乳酸试剂 盒测定细胞培养液中的乳酸值，从而判断二甲双股对高糖下 细胞的影响。

\section{3 统计学方法}

统计学分析采用 SPSS25.0 统计软件进行数据处理, 样 本均数采用样本 $\mathrm{t}$ 检验, 多样本均数采用单因素方差分析, 以 $\mathrm{P}<0.05$ 具有统计学意义。

\section{3 结果与分析}

\section{1 探究最适宜高糖胁迫 LO2 肝细胞的 G 的浓度}

在进行此实验后探究了最适宜高糖胁迫 $\mathrm{LO} 2$ 肝细胞的 $\mathrm{G}$ 的浓度, 根据表 1 得出这样的结论: 当 $\mathrm{G}$ 浓度为 $31 \mathrm{mmol} / \mathrm{L}$ 时, 是最适宜的实验浓度。 
表 1 选取最适合高糖浓度（正常肝细胞）

\begin{tabular}{|c|c|c|c|c|c|c|c|}
\hline $\begin{array}{c}\text { 加人葡萄糖的 } \\
\text { 浓度 }(\mathrm{mmol} / \mathrm{L})\end{array}$ & 25 & 27 & 29 & 31 & 33 & 35 & 0 \\
\hline $\begin{array}{c}\text { 存活肝细胞数 } \\
\text { 目 } 12 \mathrm{~h} \text { (个) }\end{array}$ & $\begin{array}{c}1.12 \times \\
10 * 7\end{array}$ & $\begin{array}{c}1.06 \times \\
10 * 7\end{array}$ & $\begin{array}{c}1.02 \times \\
10 * 7\end{array}$ & $\begin{array}{c}0.98 \times \\
10 * 7\end{array}$ & $\begin{array}{c}0.88 \times \\
10 * 7\end{array}$ & $\begin{array}{c}0.40 \times \\
10 * 7\end{array}$ & $\begin{array}{c}1.30 \times \\
10 * 7\end{array}$ \\
\hline $\begin{array}{c}\text { 存活肝细胞数 } \\
\text { 目 } 13 \mathrm{~h} \text { (个) }\end{array}$ & $\begin{array}{c}2.04 \times \\
10 * 7\end{array}$ & $\begin{array}{c}1.97 \times \\
10 * 7\end{array}$ & $\begin{array}{c}1.95 \times \\
10 * 7\end{array}$ & $\begin{array}{c}1.92 \times \\
10 * 8\end{array}$ & $\begin{array}{c}1.72 \times \\
10 * 8\end{array}$ & $\begin{array}{c}1.25 \times \\
10 * 8\end{array}$ & $\begin{array}{c}2.60 \times \\
10 * 7\end{array}$ \\
\hline 存活肝细胞数 & $3.99 \times$ & $3.92 \times$ & $3.79 \times$ & $3.76 \times$ & $3.63 \times$ & $3.19 \times$ & $5.20 \times$ \\
目 $14 \mathrm{~h}($ 个 $)$ & $10 * 7$ & $10 * 7$ & $10 * 9$ & $10 * 9$ & $10 * 9$ & $10 * 9$ & $10 * 7$ \\
\hline 存活肝细胞数 & 1.68 & 2.46 & 3.27 & 3.48 & 4.63 & 5.34 & 0 \\
目 $15 \mathrm{~h}$ (个) & \pm 0.29 & \pm 0.79 & \pm 1.38 & \pm 1.42 & \pm 1.63 & \pm 2.26 & 0 \\
\hline 存活肝细胞数 & 3.89 & 4.79 & 5.24 & 5.89 & 6.23 & 8.98 & 0 \\
目 $16 \mathrm{~h}($ 个 $)$ & \pm 1.42 & \pm 1.68 & \pm 2.17 & \pm 2.64 & \pm 2.79 & \pm 2.86 & 0 \\
\hline 存活肝细胞数 & 6.72 & 8.43 & 9.14 & 9.28 & 10.87 & 11.27 & 0 \\
目 $17 \mathrm{~h}($ 个 $)$ & \pm 2.33 & \pm 2.56 & \pm 2.99 & \pm 3.11 & \pm 3.34 & \pm 3.39 & 0 \\
\hline
\end{tabular}

\section{2 二甲双胍对高糖胁迫下正常肝细胞乳酸代谢的}

\section{影响}

在培养基中配置 $30 \mathrm{mmol} / \mathrm{L}$ 二甲双胍, 设置对照组与实 验组, 分别设计了正常肝细胞、高糖 + 正常肝细胞、二甲双 胍 + 正常肝细胞、高糖 + 二甲双胍 + 正常肝细胞, 在培养的 $12 \mathrm{~h} 、 24 \mathrm{~h} 、 48 \mathrm{~h}$ 所测得的乳酸浓度变化如表 2 所示。

\section{表 2 二甲双胍对高糖胁迫下正常肝细胞乳酸代谢的影响}

\begin{tabular}{|c|c|c|c|c|c|c|}
\hline & \multicolumn{3}{|c|}{ 第一组（正常组肝细胞） } & \multicolumn{3}{|c|}{ 第二组 (正常组 + 高糖 ) } \\
\hline & $12 \mathrm{~h}$ & $24 \mathrm{~h}$ & $48 \mathrm{~h}$ & $12 \mathrm{~h}$ & $24 \mathrm{~h}$ & $48 h$ \\
\hline $\begin{array}{c}\text { 加入葡萄糖的浓 } \\
\text { 度 } \mathrm{mmol} / \mathrm{L}\end{array}$ & \multicolumn{3}{|c|}{ I } & \multicolumn{3}{|c|}{31} \\
\hline $\begin{array}{l}\text { 加人二甲双胍浓 } \\
\text { 度 }(\mathrm{mmol} / \mathrm{L})\end{array}$ & \multicolumn{3}{|c|}{ I } & \multicolumn{3}{|c|}{ I } \\
\hline $\begin{array}{l}\text { 乳酸浓度 } \\
(\mathrm{mmol} / \mathrm{L})\end{array}$ & 0 & 0 & 0 & $\begin{array}{c}3.48 \\
\pm 1.42\end{array}$ & $\begin{array}{l}5.89 \\
\pm 2.64\end{array}$ & $\begin{array}{l}9.28 \\
\pm 3.11\end{array}$ \\
\hline $\begin{array}{c}\text { 存活肝细胞数目 } \\
\text { (个) }\end{array}$ & $\begin{array}{l}1.30 \times \\
10 * 7\end{array}$ & $\begin{array}{c}2.60 \times \\
10 * 7\end{array}$ & $\begin{array}{l}5.20 \times \\
10 * 7\end{array}$ & $\begin{array}{c}0.98 \times \\
10 * 7\end{array}$ & $\begin{array}{c}1.92 \times \\
10 * 8\end{array}$ & $\begin{array}{c}3.76 \times \\
10 * 9\end{array}$ \\
\hline & \multicolumn{3}{|c|}{ 第三组 $\underset{\text { 双胍 })}{\text { 正常组 }+ \text { 二甲 }}$} & \multicolumn{3}{|c|}{$\begin{array}{c}\text { 第四组（肝细胞 + 高糖 + } \\
\text { 二甲双胍） }\end{array}$} \\
\hline & $12 \mathrm{~h}$ & $24 \mathrm{~h}$ & $48 \mathrm{~h}$ & $12 \mathrm{~h}$ & $24 \mathrm{~h}$ & $48 \mathrm{~h}$ \\
\hline $\begin{array}{c}\text { 加入葡萄糖的浓 } \\
\text { 度 } \mathrm{mmol} / \mathrm{L}\end{array}$ & \multicolumn{3}{|c|}{ I } & \multicolumn{3}{|c|}{31} \\
\hline $\begin{array}{l}\text { 加人二甲双胍浓 } \\
\text { 度 }(\mathrm{mmol} / \mathrm{L})\end{array}$ & \multicolumn{3}{|c|}{30} & \multicolumn{3}{|c|}{30} \\
\hline $\begin{array}{l}\text { 乳酸浓度 } \\
(\mathrm{mmol} / \mathrm{L})\end{array}$ & 0 & 0 & 0 & $\begin{array}{c}3.30 \\
\pm 1.39\end{array}$ & $\begin{array}{l}5.83 \\
\pm 2.63\end{array}$ & $\begin{array}{l}9.17 \\
\pm 3.08\end{array}$ \\
\hline $\begin{array}{c}\text { 存活肝细胞数目 } \\
\text { (个) }\end{array}$ & $\begin{array}{l}1.30 \times \\
10 * 7\end{array}$ & $\begin{array}{c}2.60 \times \\
10 * 7\end{array}$ & $\begin{array}{c}5.20 \times \\
10 * 7\end{array}$ & $\begin{array}{c}1.15 \times \\
10 * 7\end{array}$ & $\begin{array}{c}2.24 \times \\
10 * 7\end{array}$ & $\begin{array}{c}4.79 \times \\
10 * 7\end{array}$ \\
\hline
\end{tabular}

根据此实验组与对照组可得出, 二甲双胍本身并不会造 成肝细胞产生孚酸, 在高糖环境下, 根据二四组的实验结果 对比可知, 在加二甲双胍前, 高糖 + 正常肝细胞 $12 \mathrm{~h}$ 内培养 液中测得的乳酸浓度值从 $1.42 \mathrm{mmol} / \mathrm{L}$ 增长到 $3.48 \mathrm{mmol} / \mathrm{L}$, $24 \mathrm{~h}$ 后最高增加至 $5.89 \mathrm{mmol} / \mathrm{L}, 48 \mathrm{~h}$ 后细胞数量增加率开始 显著性下降, 乳酸浓度也增加至 $9.289 \mathrm{mmol} / \mathrm{L}$, 在加人二甲 双胍后, 从结果上看乳酸浓度和细胞数量与仅高糖胁迫下的 结果比较并无显著的变化。

\section{4 讨论}

大量研究表明，乳酸酸中毒是糖尿病患者一种少见而严 重的并发症，大多发生在使用双胍类药物并伴随有肝肾功能 不全、心力衰竭等患者中 ${ }^{[9,10]}$ 。近年研究认为, 因使用正常治 疗剂量的二甲双胍导致的相关性乳酸酸中毒 (Metformin Lactate Acidosis，MALA ) 很罕见，但如果临床使用不当也可能 导致血浆乳酸含量升高, 甚至导致乳酸酸中毒 ( Lactate Acidosis, LA ) 发生 ${ }^{[11]}$ 。肝脏是葡萄糖代谢的重要器官, 肝脏能摄 取和利用葡萄糖降低血糖, 并能将葡萄糖转化为肝糖原储存 起来。肝硬化的患者胰岛素抵抗加重, 会影响糖代谢而引起 肝源性糖尿病。而糖尿病也会影响肝脏, 尤其是 2 型糖尿病 患者, 容易出现肝功能损害以及非酒精性脂肪肝 ${ }^{[12]}$ 。

二甲双胍作为传统的降糖药，能促进葡萄糖的代谢，增 加其无氧酵解，提高了乳酸水平从而导致乳酸酸中毒; 此外 二甲双胍由于能抑制肝脏和肌肉对乳酸的利用, 抑制糖异生, 从而减少葡萄糖的生产, 进而使乳酸堆积增加了发生乳酸中 毒的风险 ${ }^{[13-15]}$ 。

本试验研究发现, 二甲双胍对高糖环境下的肝细胞乳酸 代谢影响不大, 实验组与对照组加入不同水平的二甲双胍后， 所测得的乳酸含量并无较大差异，但不同水平的二甲双胍可 促进细胞增殖。高糖环境能抑制肝细胞的增殖，其原因可能 是高糖诱导肝细胞中 STC2 表达, 而过量表达的 STC2 能进 一步增强高糖所诱导的肝细胞增殖抑制能力 ${ }^{[16]}$ 。另外, 也有 研究表明高糖能促进 TNF - $\alpha$ 、IL - 6、等炎性细胞因子分泌, 调控调亡相关分子 B 淋巴瘤 2 和 Bax 表达，从而诱导肝细胞 凋亡 ${ }^{[17]}$ 。二甲双胍能促进肝细胞增殖, 可能是因为二甲双胍 可以通过 AMPK 依赖途径等途径，抑制炎性细胞因子的分 泌及核转录因子 $-\kappa \mathrm{B}(\mathrm{NF}-\kappa \mathrm{B})$ 活性, 从而达到促进细胞 增殖的目的 ${ }^{[18]}$ 。

综上所述，二甲双胍对高糖环境下的肝细胞乳酸代谢情 况无太大影响, 但不同浓度的二甲双胍对肝细胞有保护机制, 可以促进细胞增殖。

\section{参考文献}

[1] 母义明, 纪立农.二甲双胍临床应用专家共识 (2016 年版) [J]. 中 国糖尿病杂志 ,2016(10):871-884.

[2] 党永岩, 叶希韵, 申杰. 葡萄糖浓度波动对人肝 L02 细胞损伤的 实验研究 [J]. 华东师范大学学报,2010(05):143-148. 
[3] 苏弘薇, 韦勋, 潘海林, 等. 2 型糖尿病患者血乳酸水平及其影响 因素 [J]. 糖尿病天下,2015(02):215-218.

[4] 宁光. 中国糖尿病防治的现状及展望 [J]. 中国科学 (生命科 学 ),2018(08):810-811.

[5] Lilian Beatriz Aguayo Rojas,Marilia Brito Gomes. Metformin: an old but still the best treatment for type 2 diabetes[J]. Lilian Beatriz Aguayo Rojas;Marilia Brito Gomes,2013(01):143-146.

[6] 张味娜, 俞敏, 孙美云, 等.二甲双胍致乳酸性酸中毒 1 例 [J]. 浙 江宁海县第一医院药剂科中国乡村医药, 2020(01):43-44.

[7] 录影萍. 特殊人群慎服二甲双胍 [N]. 上海中医药报, 2019 .

[8] 林健, 周智广. 糖尿病乳酸酸中毒的诊断治疗及进展 $[\mathrm{J}]$. 临床内科 杂志 ,2017(34):159-161.

[9] Richy FF,Sabido-Espin M,Guedes S,et al. Incidence of lactic acidosis in patients with type 2 diabetes with and without renal impairment treated with metformin: a retrospective cohort study $[\mathrm{J}]$.Diabetes Care,2014(08):2291-2295.

[10] Aharaz A,Pottegard A,Henriksen DP,et al. Risk of lactic aci- dosis in type 2 diabetes patients using metformin: A case control study[J]. PloS One, 2018(05)196+122.

[11] Xianbin Cai,Xi Hu,Bozhi Cai,et al.Metformin suppresses hepatocellular carcinoma cell growth through inductionof cell cycle G1/G0 phase arrest and p21CIP and p27KIP expression and downregulationof cy- clin D1 in vitro and in vivo[J]. Oncology Reports,2013(05):438-441.

[12] 郭雅丽, 卢书明, 李春艳, 等.肝硬化并糖尿病患者胇岛素抵抗及 与肝功能关系探讨 [J]. 大连医科大学学报 ,2017(04):378-382.

[13] Almirall J, Briculle M, Gonzalez clemente JM. Metformin-as-sociated lactic acidosis in type 2 diabetes mellitus : incidence and presentation in common elinical practice[J]. Nephrol Dial Trans-plant, $2008(23): 2436-2438$.

[14] 陆祖谦. 双胍类降糖药物对 2 型糖尿病治疗价值的重新认识 [J]. 临床药物治疗杂志 ,2010(04):33-38.

[15] McAlister FA, Majumdar SR, Eurich DT et al.The effect of specialist care within the first year on subsequent outcomes in 24232 adults with new-onset diabetes mellitus:population based cohort study [J].Qual Saf Health Care,2007(01):6-11.

[16] 陶文玉, 陈郊丽, 李晓进, 等. 斯钻素 2 在高糖诱导的肝细胞炎症 反应中的作用机制 [J]. 实用医学杂志 ,2019(14):2220-2224+2229.

[17] 李妍晨, 黄才斌, 刘瑶, 等. 高迁移率族蛋白 B1 在高糖微环境诱 导人肝细胞恶性转化过程中作用和机制的研究 [J]. 中国糖尿病杂 志 $, 2019(02): 137-142$

[18] Li Z, Ding Q, Ling LP, et al.Metformin attenuates motility, contraction, and fibrogenic response of hepatic stellate cells in vivo and in vitro by activating amp-activated protein kinase[J].World J Gastroenterol, 2018(07) :819-832. 